CredibleMeds.org: What does it offer?

\author{
Raymond L. Woosley, MD, PhD ${ }^{1,2}$ \\ Kristin Black, B.S. ${ }^{2}$ \\ Craig W. Heise, $\mathrm{MD}^{1}$ \\ Klaus Romero, MS, $\mathrm{MD}^{3}$
}

1. Division of Clinical Data Analytics and Decision Support Department of Medicine, College of Medicine-Phoenix University of Arizona, Phoenix, Arizona 85004-2157

2. AZCERT, Oro Valley, Arizona 85755

3. Critical Path Institute, Tucson Arizona 85718 


\section{Introduction to CredibleMeds.org}

The CredibleMeds.org website is a unique resource for anyone with a question regarding the QT interval and the many drugs known to prolong QT and cause torsades de pointes (TdP). However, the site offers much more than just a list of drugs. The website was launched in 2001 as an educational resource by the Arizona Center for Education and Research on Therapeutics (aka AZCERT and ArizonaCERT). AZCERT was one of fourteen federally funded centers in a network created by the Agency for Healthcare Research and Quality (AHRQ) to improve health outcomes from therapeutics by conducting coordinated research and educational programs that focused on various aspects of therapeutics. AZCERT, initially based at the University of Arizona, focused on drug-drug interactions that were responsible for QT prolongation and TdP. In 2012, AZCERT transitioned from a university-based program to a freestanding non-profit 501c3 corporation funded by the US Food and Drug Administration, research grants and charitable contributions. AZCERT and its CredibleMeds website are recognized as the singular international resource for providing reliable, up to date and independent information on medications, especially those associated with QT prolongation and/or TdP. To prevent real or perceived conflicts of interest and to assure independence in the decision-making process for assessing TdP causality, AZCERT has been entirely supported by peer-reviewed federal awards and charitable public contributions.

\section{What Credible Meds.org Offers}

Most search engines when queried with "QTdrugs", "torsades.org" or "AZCERT", quickly direct visitors to the CredibleMeds.org home page where visitors can easily search the four 
QTdrugs lists of drugs with different levels of risk of TdP by simply entering a drug name. If the visitor wishes to print or download the complete lists of drugs, the site requires the visitor to register so that they can be notified by email any time the lists are revised. This is considered necessary for the safe use of the lists due to the fact that they are revised every 4-8 weeks.

The home page for the website offers visitors three main portals shown in figure 1. All three offer access to the QTdrugs lists but each has additional information that is tailored either "For Everyone", For Healthcare Providers" or "For Research Scientists." The first link on the navigation bar for the three portals is "QTdrugs Lists (registration required). Unregistered visitors who seek access to the full QTdrugs lists are directed to a registration page and registered users are taken directly to the lists.

\section{For Everyone - QTdrugs Lists (Registration Required)}

Under a contract with the U.S. Food and Drug Administration's Safe Use Initiative, AZCERT maintains the website and the Lists of drugs that cause TdP and/or prolong QT. The lists were initially named and are still generally known as the QTdrugs lists(1). The continually updated lists are provided free to over 95,000 registered visitors from 193 countries. Forty five ???percent of registrants are healthcare providers, $40 \%$ are persons and $15 \%$ are research scientists. A survey of visits found that $95 \%$ had a positive impression of the website. (Postema)

To make ready access to the lists possible, AZCERT has developed and released an application program interface (API) that enables health information technology systems to have online open-source access to the lists. Also, the lists can be searched using smartphone apps that are available free from the Apple Store, Google Play or Microsoft Mobile. 
Medications are evaluated for their risk of causing QT prolongation or TdP by a Scientific Review Committee with three physician members (RLW, CWH, KR). The committee's recommendations for placement of a drug into a TdP risk category are reviewed by an International Advisory Board of 39 recognized authorities on drug safety and cardiovascular medicine (https://www.crediblemeds.org/research-scientists/advisory-board/).

The process used by AZCERT to analyze evidence and assess TdP causality for drugs is described on the CredibleMeds website (ㅁ) and in peer-reviewed publications (16,34,drug safety) as the Adverse Drug Event Causality Analysis (ADECA). Because the available evidence frequently has limitations and gaps, and is often of variable quality, AZCERT uses this systematic, in-depth analysis of all available data to assess causality. Since the lists were first compiled and posted on the internet in 1999, over 135 new drugs have been added and many have been moved among categories or removed. Beginning in 2012, many drugs marketed outside the United States, especially in Europe, Japan and Canada, have been reviewed and some have been added to the lists with designation as marketed outside the United States.

The risk categorization process begins when suspect drugs are brought to the attention of AZCERT scientists, usually in 1 of the 3 ways shown at the top of Figure 2 . The professional staff assess the ability of each drug to cause TdP by analyzing the available laboratory and clinical evidence and applying the Bradford Hill criteria for causality (i.e., the conditions necessary to provide adequate evidence of a causal relationship between incidence and a consequence) (4). The analysis includes a thorough review of published studies (PubMed search using standardized search terms) and all adverse events reported to the FDA's Adverse Event Reporting System (AERS), and uses the empirical Bayesian data-mining statistical software 
developed for the FDA (Empirica Signal Software, Oracle Health Sciences, Redwood Shores, California) (16).

CredibleMeds places drugs in 4 categories of TdP risk. Drugs with "known risk of TdP" are those found to have convincing evidence that they can cause TdP, even when administered as recommended in the drug's FDA-approved label. Drugs that prolong the QT interval during routine clinical use, but do not at this time have convincing evidence of TdP causality, are classified as having a "possible risk of TdP." Beginning in 2006, the "conditional risk" category was added and includes drugs for which there is a risk of TdP, but only under certain specific conditions, such as overdose, hypokalemia, hypomagnesemia, bradycardia, or when there is an interaction with another drug(s). This category also includes drugs that are associated with TdP due to their ability to create the conditions that enable another drug to cause TdP (e.g.. loop diuretic agents or metabolic inhibitors of a QT-prolonging drug). The website also posts a fourth list of drugs that should be avoided by patients with congenital long QT syndrome (CLQTS), when it is clinically feasible. Because the consequences for patients with CLQTS could be lethal, this list is generated by combining all of the drugs in the 3 risk categories listed previously and adding the adrenergic drugs (Special Risk Category) that are considered to place some CLQTS patients at high risk of sudden death (16).

The process for evaluating drugs and all decisions regarding their inclusion on lists are overseen by an international Advisory Board of clinical and pharmacological experts. The Board's advice is also sought whenever conflicting evidence must be resolved or when any Board member expresses concern about a decision made by the Scientific Review Committee. Medications on all four lists are continuously monitored for new evidence and, in recent years, 
the lists have been revised every 4 to 8 weeks. Figure 3 shows how the number of drugs on each list has changed since they were first launched in 1999. Currently, 57 medications are on the list of drugs known to cause TdP (including 9 removed from the U.S. market, but which may still be available in some countries). Another 91 are on the list for possible risk of TdP and 43 are on the list for conditional TdP risk. Two hundred and twenty three medications are on the list of Drugs to Avoid by patients with CLQTS. Figure 4 shows the broad range of therapeutic areas for the drugs on the QTdrugs lists and demonstrates the breadth of medical impact. CredibleMeds has become an invaluable tool for the clinical management of patients affected by CLQTS (9). Established arrhythmia centers caring for these patients refer them to the CredibleMeds website so they or a family member can print an updated list of drugs to avoid (9). Since many general practitioners and even cardiologists are unaware of the QTprolonging potential of many cardiac and noncardiac drugs, (refs) this practice has become an essential part of the management of patients with channelopathies.

The visitor to CredibleMeds has several options for how to download or print the information in the QTdrugs list. Each list can be copy/pasted or printed as a table that includes each drug's generic name, most common brand names, Drug Class, Therapeutic Use, TdP Risk Category and Route of Administration. The list can also be converted to a PDF or Excel File and printed. The user can also print a shorter 2-3 page PDF in English or Spanish that lists only the drug names (generic and brand) and risk category. In the last year, these PDFs have been downloaded and printed 32,000 times with $15 \%$ of those in Spanish. The shorter format is available as a "Combined List" of drugs in the Known, Possible and Conditional risk categories or as the longer list of all categories for Drugs to Avoid by patients with CLQTS. 


\section{For Everyone - Create “My Medicines" Online Lists}

To address one of the most challenging aspects of therapeutics, AZCERT has created an online repository for patients to create and maintain a list of the medicines they are taking. The form prompts the user to remember to include hormones, vitamins, non-prescription medicines and dietary supplements. When creating the list, the program informs the user if any drug entered is on the QTdrugs lists and later informs the user if one of the drugs they are taking has been newly added to the lists. The lists can be printed or emailed for delivery to the patient's pharmacists or physician's office.

\section{For Everyone - Educational Tools}

Several links are on the navigation bar that take the visitor to 1) Educational Papers written for the lay person, 2) a Guide for Safe Medication Use, 3) a Drug Interaction Advisory on the 25 major drug-drug interactions, 4) useful links to other reliable sources of information on medicines and 5) a list of scientific peer-reviewed articles published by AZCERT scientists.

\section{For Everyone - Virtual Medicine Cabinet}

The Virtual Medicine Cabinet is an educational tool designed as a cartoon drawing of a medicine cabinet filled with the non-prescription medicines that are commonly found in the home. By clicking on any of the products in the cabinet, the user will see a pop-up that alerts them to potentially dangerous interactions relevant to these non-prescription drugs.

\section{For Healthcare Providers - Rx Tools - Flockhart's Table}


This link takes the visitor to several educational articles and tools important to the prescriber. Downloadable monographs developed in collaboration with the American Medical Association are available on topics such as Pharmacogenetics and Warfarin Genetic Testing. A Powerpoint educational module on Preventable Adverse Drug-Drug Interactions that was developed in collaboration with the FDA has been downloaded over 50,000 times. Articles on drug-induced long QT, short QT syndrome and methadone drug interactions are available. A link is provided to Indiana University's Cytochrome P450 Clinically Relevant Table of Drug-Drug Interactions that was developed by Dr. David Flockhart, a founding member of the AZCERT Board.

Flockhart's table is an internationally recognized resource to understand and predict drug-drug interactions.() A link is also to Frequently Asked Questions (FAQs) that are relevant to the interests and issues of prescribers.

\section{For Healthcare Providers - OncoSupport for Oncology Prescribing}

This link in the navigation bar takes the visitor to a table listing the drugs commonly prescribed by oncologists that are in one of the three TdP risk categories. These includes anti-cancer drugs, anti-nausea medicines, antidepressants and anesthetics. Similar tables are being developed for neuropsychiatry and internal medicine.

\section{For Research Scientists}

This portal includes a link that takes researchers to a full description of the ADECA process that is used to evaluate evidence in creating the QTdrugs Lists. There is a link to a page for the Scientific Review Committee and the Advisory Board which includes photos and brief bios for its 39 members. Another link goes to a list of over 90 peer-reviewed publications that have 
culminated from the research and educational programs in AZCERT. This portal also includes referenced articles that describe how the QTdrugs lists have been used in cardiovascular epidemiology research and in quality improvement programs that are based on clinical decision support systems.

\section{Role of CredibleMeds in Decision Support Systems}

The overall goal of the CredibleMeds website is to foster and support efforts to prevent TdP. Consensus papers have defined what is required for prevention of TdP but success requires that physicians be able to manage massive amounts of information about the drugs and their patients. This has prompted scientists to develop Clinical Decision Support Systems (CDSS) to help physicians and other members of the health care team to reach optimal medical and therapeutic decisions that reduce the risk of TdP. Using the QTdrugs lists as part of a CDSS, investigators at the Mayo Clinic (49) and the University of Indiana issue alerts that warn of potential harm from a prescribing decision $(52,67,68)$. These programs have been shown to improve prescribing and reduce the risk of QT prolongation in hospitalized patients. Unfortunately, the beneficial impact of these CDSS has been limited because in $75 \%$ to $95 \%$ of cases, physicians and pharmacists override or ignore the alerts $(50,52,69)$ and no CDSS has yet been found to have sufficient impact on prescribing to reduce the incidence of TdP. To improve the acceptance of alerts and the effectiveness of CDSS overall, an expert panel has recommended that CDSS should only provide clinically relevant and essential information at the time when a decision is being made, without overwhelming the decision makers with often annoying irrelevant alerts (70). Moving away from the use of alerts to signal prescribing errors, the concept of "medical autopilots" has been suggested as a preferred approach for CDSS (71). 
Such a program in now in place in hospitals affiliated with the University of Arizona which monitors the EMR and sends advisories to guide prescribers toward decisions that result in maximum benefit and minimal risk of TdP. Figure 5 shows the four types of advisories that can be issued and tailored to the risk profile of the patient. Hopefully, in the near future, a CDSS with these characteristics will be shown to prevent TdP by incorporating the QTdrugs lists and all of the clinical and genomic risk factors that we know can contribute to a person's risk of TdP.

\section{References}

1. Romero K, Woosley RL. Clarification to the www.qtdrugs.org updated lists. Pharmacoepidemiol Drug Saf 2009;18:423-4.

2. Woosley RL, Romero KA. www.Crediblemeds.org QTdrugs List, 2016, AZCERT, Inc. Available at: https://crediblemeds.org. Accessed January 22, 2016.

3. CredibleMeds Process for Evaluating Evidence and Assigning Risk. Available at: https://www.crediblemeds.org/research-scientists/why-lists/. Accessed January 22, 2016.

4. Hill AB. The environment and disease: association or causation? Proc R Soc Med $1965 ; 58: 295-300$.

5. Postema PG, Neville J, de Jong JS, et al. Safe drug use in long QT syndrome and Brugada syndrome: comparison of website statistics. Europace 2013;15:1042-9.

\section{Figure Legends}


Figure 1. The three major portals for CredibleMeds (For Everyone, For Healthcare Providers and For Research Scientists) and the navigation bars that are available at each page.

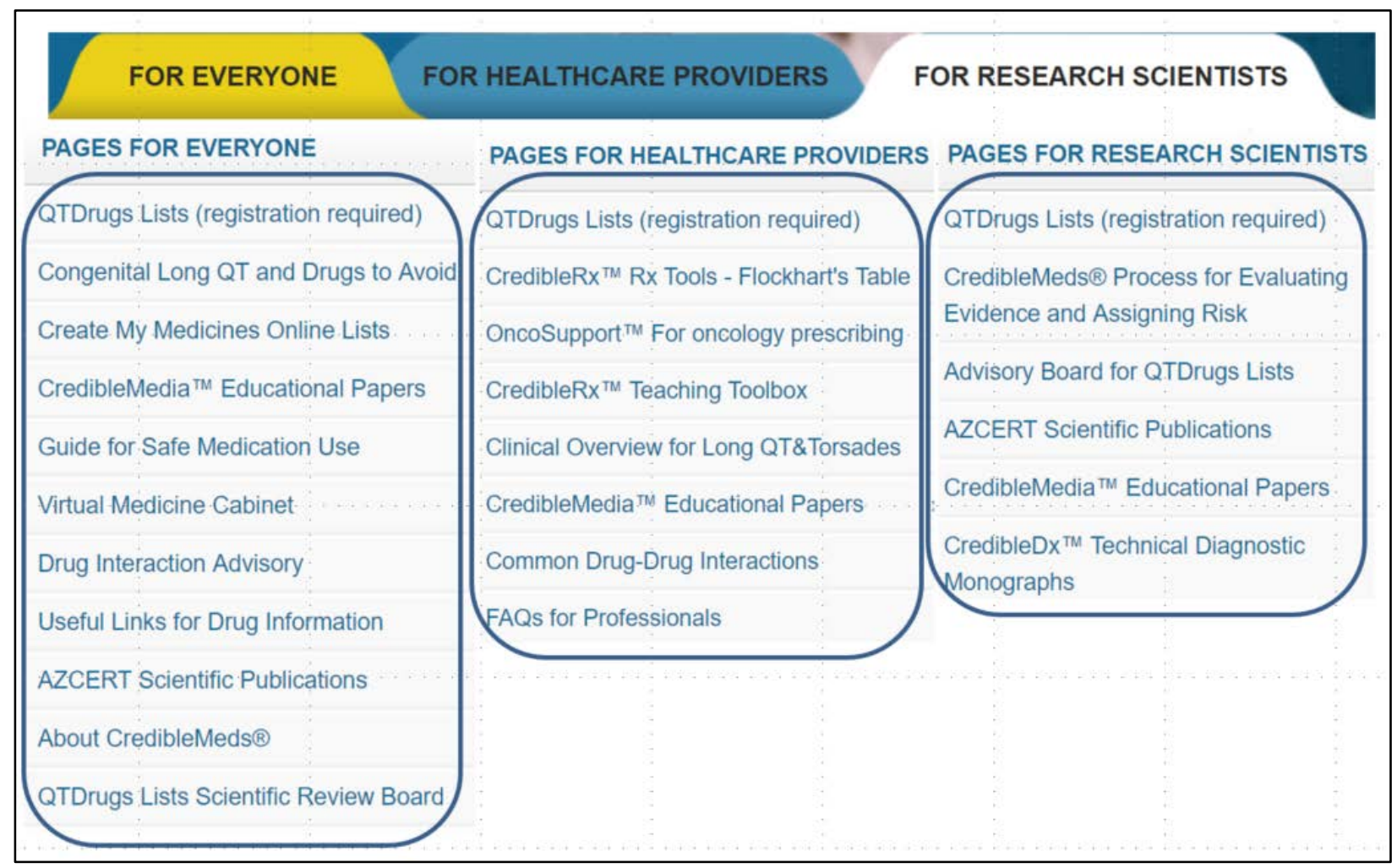


Figure 2: Schematic for the ADECA Causality Analysis of Relative Drug Safety

Drugs from 1 or more of the 3 primary sources (cases, the medical literature, or drug labeling for new or previously marketed drugs) are evaluated for their risk of QT prolongation, TdP, or both. The Bradford Hill criteria (31) are used to analyze all available evidence and construct a summary report that is reviewed by the CredibleMeds.org team and, in some cases, referred to the Advisory Board for consideration. On the basis of this analysis, an iterative process is used to decide whether the drug should be placed on 1 of the 3 lists: drugs with TdP risk; drugs with possible TdP risk; or drugs with conditional TdP risk. All drugs on these lists continue to be monitored for new evidence that could change their classification. From Woosley and Romero (16), with permission. FDA = Food and Drug Administration; TDP = torsades de pointes.

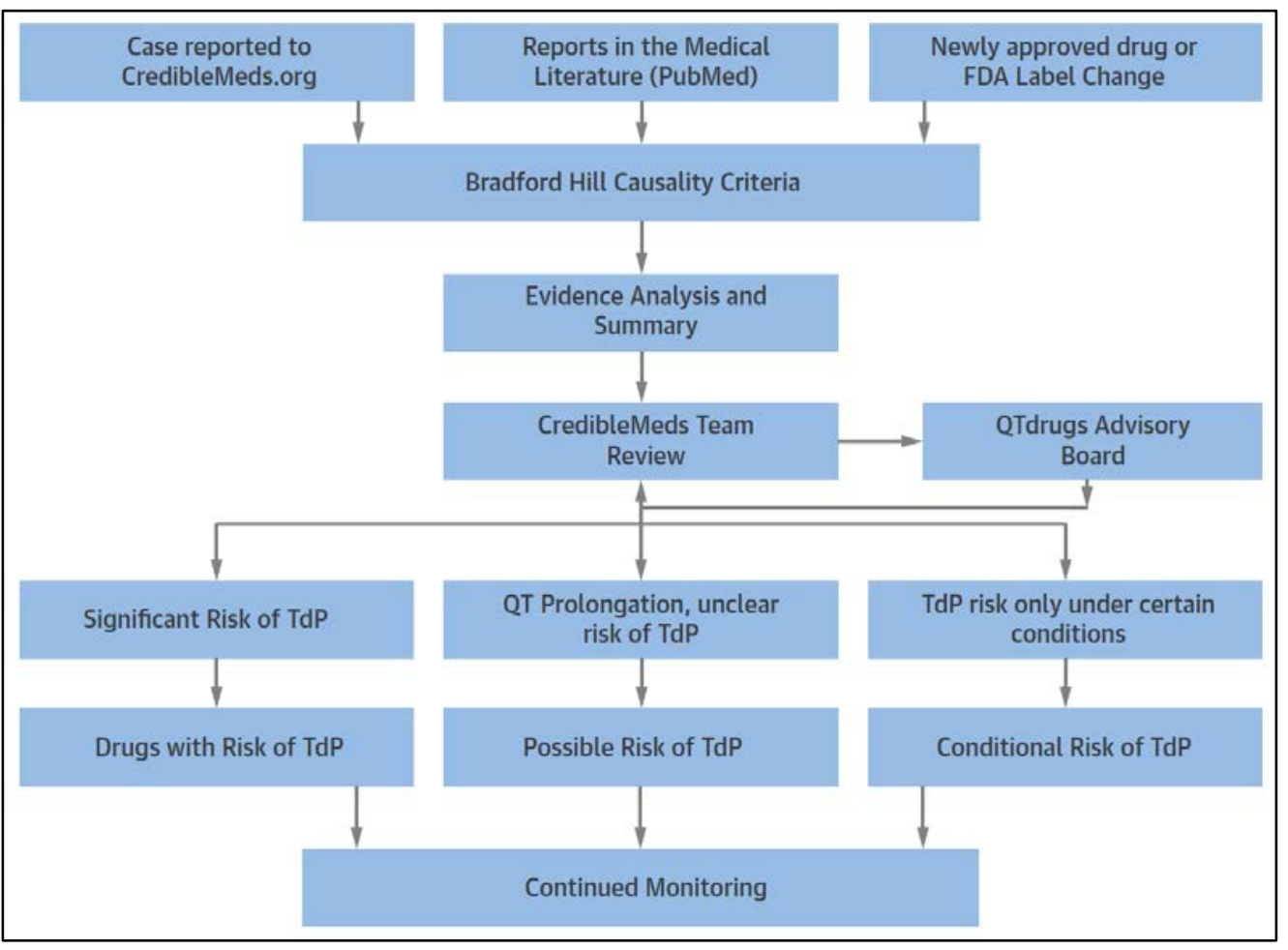


Figure 3: The Number of Drugs in Each of the 4 Categories of TdP Risk on the CredibleMeds website from 1999 Trough 2017

The graph displays the changing number of drugs in each of the four categories of TdP risk from the initial postings at www.CredibleMeds.org in 1999 until January 2015. The number of drugs on the list of drugs with Known Risk of TdP at any point in time are shown as a green line. The number of drugs with Possible Risk of TdP are shown in red. The number of drugs with Conditional Risk of TdP (i.e. associated with TdP under certain conditions) are shown in orange. The number of drugs on the list of Drugs to Avoid in patients with congenital long QT Syndrome are shown in blue. The latter list includes all of the drugs listed in the other three risk categories plus other drugs that do not prolong QT per se (e.g. adrenergic drugs) but have actions known to place some patients with cLQTS at risk.

CLQTS = congenital long QT syndrome; TDP = torsades de pointes.

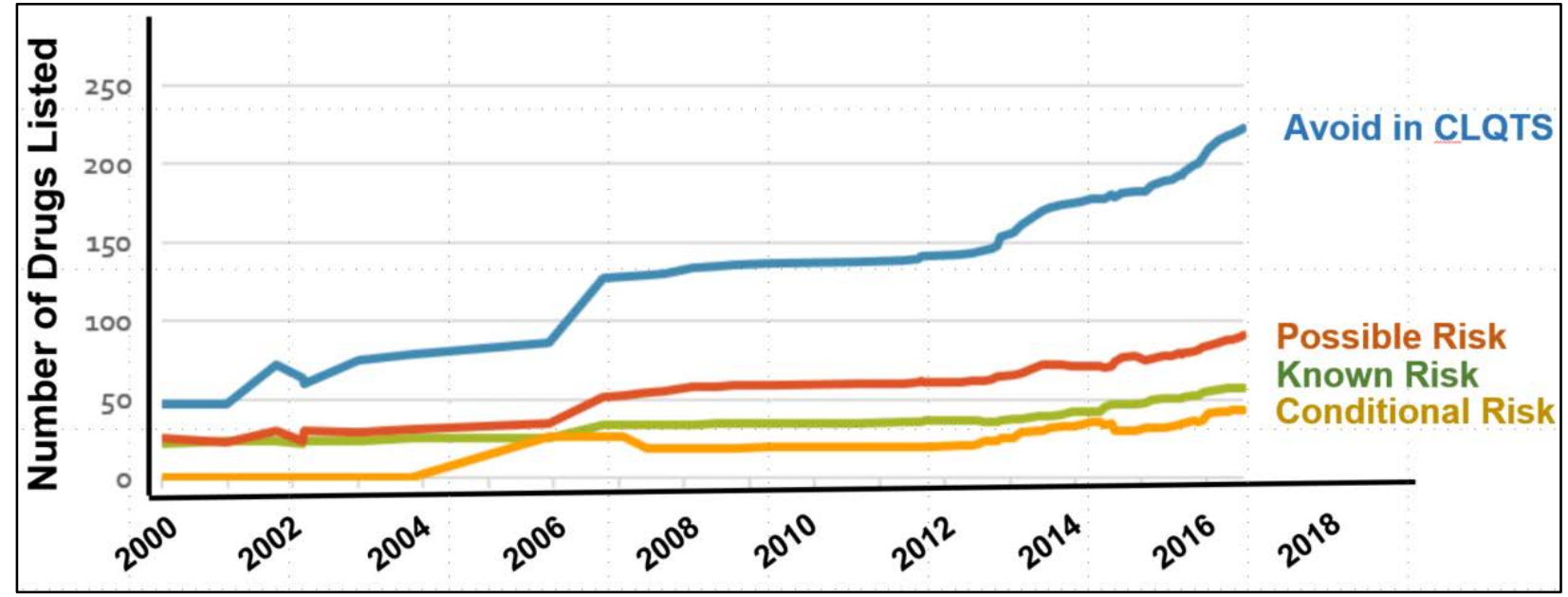


Figure 4: Spectrum of Therapeutic Areas for Drugs Listed at www.CredibleMeds.org With

\section{Known Risk of TdP, Possible Risk of TdP and Conditional Risk of TdP}

Spectrum of therapeutic areas for drugs listed at www.CredibleMeds.org with known risk of TdP, possible risk of TdP and conditional risk of TdP. The $\mathrm{X}$ axis displays the number of drugs that were in each of the therapeutic categories listed on the $Y$ axis as of July 2016. Each drug was placed in the therapeutic category in which it has demonstrated predominant clinical efficacy.

ADHD = attention deficit hyperactivity disorder; Misc. = miscellaneous; TDP = torsades de pointes.

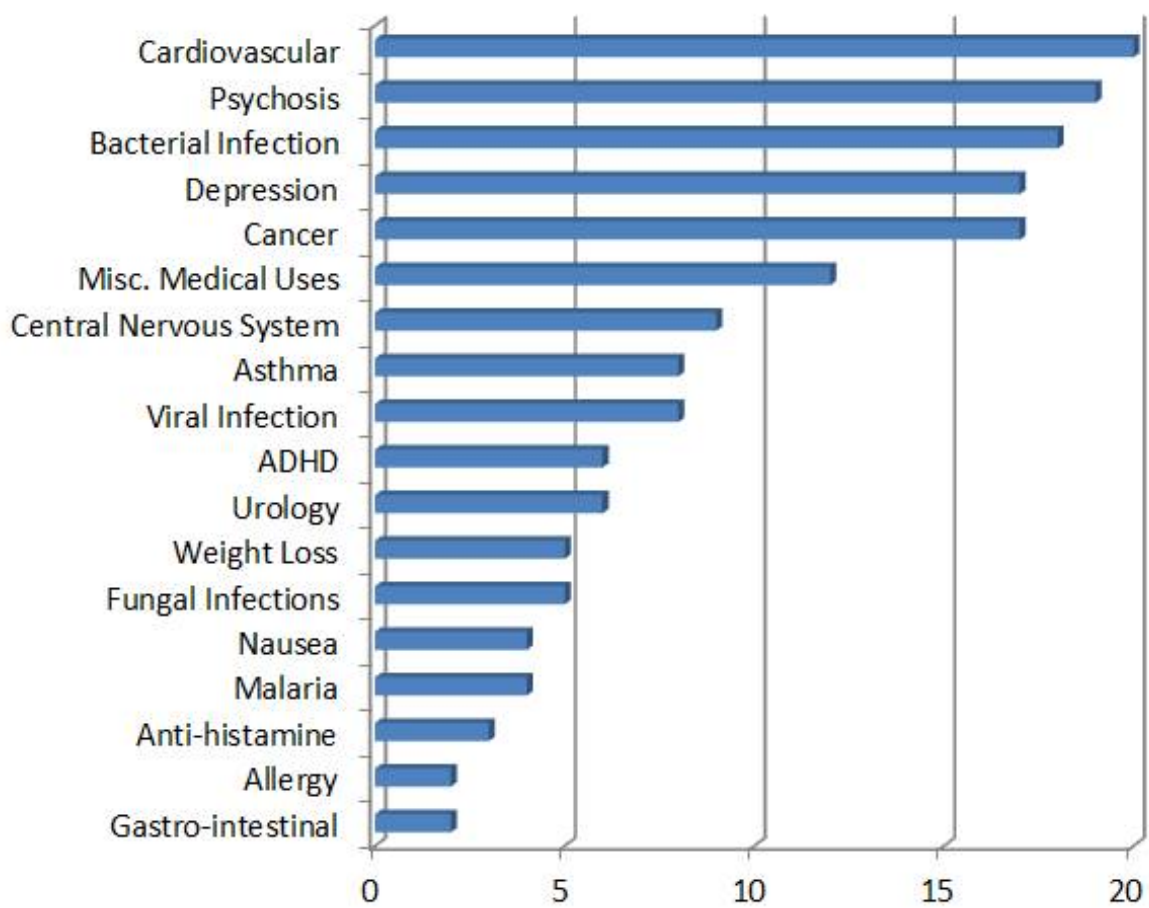


Figure 5????Maybe use this: Logic diagram for CERNER Alerts in Banner or something else from the CDSS at Banner

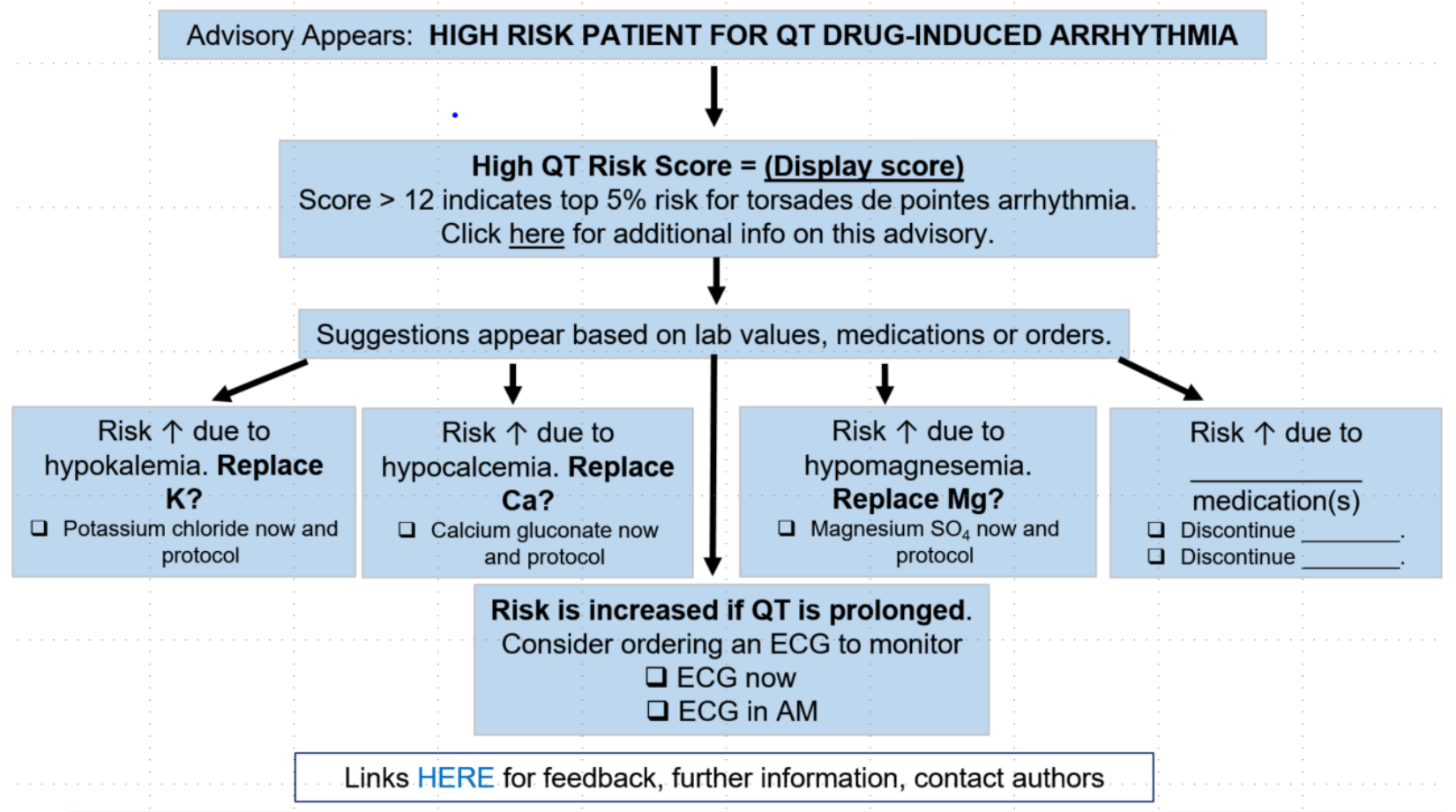


